OPEN ACCESS

Edited by:

Andrew Charles Cuming,

University of Leeds,

United Kingdom

Reviewed by:

Caspar Christian Cedric Chater,

University of Sheffield,

United Kingdom

Hanna Hõrak,

University of Tartu, Estonia

*Correspondence:

Els J. M. Van Damme

elsjm.vandamme@ugent.be

${ }^{+}$ORCID:

Guy Smagghe

orcid.org/0000-0001-8334-3313

Specialty section:

This article was submitted to

Plant Abiotic Stress,

a section of the journal

Frontiers in Plant Science

Received: 15 November 2019

Accepted: 25 March 2020

Published: 17 April 2020

Citation:

Dubiel M, Beeckman T, Smagghe $G$ and Van Damme EJM (2020)

Arabidopsis Lectin EULS3 is Involved in ABA Signaling in Roots.

Front. Plant Sci. 11:437.

doi: $10.3389 /$ fpls.2020.00437

\section{Arabidopsis Lectin EULS3 Is Involved in ABA Signaling in Roots}

\author{
Malgorzata Dubiel ${ }^{1,2}$, Tom Beeckman ${ }^{3,4}$, Guy Smagghe ${ }^{2 \dagger}$ and Els J. M. Van Damme ${ }^{1,5 *}$ \\ ${ }_{1}^{1}$ Laboratory of Biochemistry and Glycobiology, Department of Molecular Biotechnology, Ghent University, Ghent, Belgium, \\ ${ }^{2}$ Laboratory of Agrozoology, Department of Plants and Crops, Ghent University, Ghent, Belgium, ${ }^{3}$ Department of Plant \\ Biotechnology and Bioinformatics, Ghent University, Ghent, Belgium, ${ }^{4}$ VIB-UGent Center for Plant Systems Biology, Ghent, \\ Belgium, ${ }^{5}$ Center for Advanced Light Microscopy, Ghent University, Ghent, Belgium
}

The Arabidopsis thaliana lectin ArathEULS3 is upregulated in particular stress conditions and upon abscisic acid (ABA) treatment. ABA is a plant hormone important for plant growth and stress responses. During stress ABA is perceived by PYR/PYL/RCAR receptors, inhibiting protein phosphatases PP2Cs thereby enabling SNRK2s kinases to start downstream phosphorylation cascades and signaling. PYL9, one of the ABA receptors was identified as an interacting partner for ArathEULS3. Promoter::GUS activity studies revealed the expression of ArathEULS3 in the central root cylinder and the cells flanking young lateral root primordia, and showed enhanced expression in root tips after ABA treatment. Transcript levels for ArathEULS3 increased after exposure to ABA and osmotic treatments. ArathEULS3 CRISPR KO mutants served as a tool to expand the knowledge on the role of ArathEULS3 in plant development. KO lines revealed a longer root system compared to WT plants, and showed reduced sensitivity to ABA, salt, and osmotic conditions. Additionally it was noted that the KO mutants had more emerged lateral roots when grown in high osmotic conditions. Together these data suggest that ArathEULS3 may be an important player in ABA responses in roots.

Keywords: EUL lectin family, abscisic acid, root, stress, CRISPR

\section{INTRODUCTION}

Plants have developed a sophisticated set of physiological responses, in which the reaction to different environmental conditions is mediated by different hormones. The plant hormone abscisic acid $(\mathrm{ABA})$ acts as a stress hormone important during drought and high salinity conditions as well as during seed maturation (Yoshida et al., 2019). However plants also maintain low concentrations of this hormone even in non-stress conditions. Plant roots are the first to sense changing soil conditions like water shortage or osmotic pressure, which is why ABA levels rise first in the roots. When ABA reaches the leaves, it causes closing of the stomatal aperture, by inducing stressresponsive genes, reducing the entry of $\mathrm{CO}_{2}$ to the leaf, and ensuring higher tolerance to dry soil conditions. A breakthrough in understanding the mechanisms behind ABA signaling was made in 2009 when Ma et al. (2009) and Park et al. (2009) discovered that the nucleocytoplasmic ABA receptors (PYR/PYL/RCAR) can inhibit type $2 \mathrm{C}$ protein phosphatases (PP2Cs) in the presence of $\mathrm{ABA}$, which in turn release the protein kinases (SNRK2s). Activated SNRK2s phosphorylate ABAresponsive transcription factors, thereby activating ABA-responsive genes. 
Lateral root formation in Arabidopsis starts in the elongation zone, where the xylem pole pericycle cells are primed by the phytohormone auxin to become a lateral root. These specifically programmed cells are called pre-branch sites. They retain their stem cell activity and have the potential to become a lateral root (Banda et al., 2019). The initiation of a lateral root takes place in the differentiation zone of the root and causes an asymmetric cell division and formation of a lateral root primordium, a process also promoted by auxin. Since the lateral root primordia are located inside the root and are surrounded by several other cell layers (endodermis, cortex, and epidermis) they need to find a way to pass through the overlying tissues. Auxin is also responsible for signaling through the cell layers in order to change their properties, such as loosening up of the cell walls in order to ensure the emergence of the new roots. However in the roots, auxin is not the only important hormone player-ABA plays an important role in influencing root architecture during drought, osmotic and salt stresses. Suberin is a hydrophobic polymer which is formed on the surface of endodermal cells (Barberon, 2017). The formation of suberin lamellae prevents the water and nutrient uptake at the endodermis level ensuring selectivity towards compounds taken up by the plant and transported through the vascular system to the leaves. In stress conditions like drought or high salinity the suberization is increased through ABA signaling. The ectopic suberin deposition occurs in young root parts and in the cortex (Barberon et al., 2016). Next to suberin deposition, in high salinity environment, $\mathrm{ABA}$ can both stimulate primary root growth as well as inhibit lateral root growth, through endodermal signaling. During water deficit ABA also causes an inhibition of lateral root initiation, a phenomenon recently described as xerobranching (Orman-Ligeza et al., 2018). Mutants defective in PYR/PYL ABA receptors turned out to be insensitive to transient $\mathrm{ABA}$ treatment and formed lateral roots. Moreover, the lateral root priming might be repressed by ABA accumulation in the basal meristem of the root tip, disrupting the oscillatory auxin responses. Interestingly, auxin plays a pivotal role in the emergence of lateral root in the direction of more water, a process called hydropatterning, which was shown to be ABA-independent (Bao et al., 2014).

Lectins are proteins, composed of one or more lectin domains, which can be linked to other protein domains (such as protein kinase, F-box or glycosyl hydrolase domains), and are able to specifically and reversibly bind to carbohydrate or glycan structures without changing their properties (Van Holle and Van Damme, 2018). Many of the nucleo-cytoplasmic, stress-inducible lectins were shown to be important for both plant development and plant immune responses. For instance, the transcripts of more than $70 \%$ of all jacalin-related lectins are upregulated after exposure of the plant to different abiotic and biotic stresses as well as phytohormone treatments, and overexpression of some jacalins resulted in plants with enhanced resistance against pathogens. In contrast to many other lectin families that occur only in some plant families, the Euonymus europaeus (EUL) related lectins are present throughout the whole plant kingdom, starting from liverworts to flowering plants, suggesting that these proteins might play a conserved role in plants. The genome of Arabidopsis thaliana contains only one EUL gene, further referred to as ArathEULS3 (Fouquaert et al., 2009). ArathEULS3 is a stress-responsive protein that locates to the nucleo-cytoplasmic compartment of the plant cell (Van Hove et al., 2011). The expression of ArathEULS3 is enhanced after hormone treatments (ABA, MeJA, Ethephon) as well as after abiotic (drought, osmotic stress), or biotic (Pseudomonas syringae) stresses (Van Hove et al., 2014). In 2014, Li et al. suggested that ArathEULS3 is involved in ABA signaling through interaction with the ABA receptor PYL9. Furthermore, Van Hove et al. (2015) described the importance of ArathEULS3 in stomatal closure. Transgenic lines with reduced ArathEULS3 expression revealed abnormal stomatal closure in the presence of ABA and were reported to be more susceptible to bacteria Pseudomonas syringae disease whereas the plants overexpressing EULS3 showed a clear reduction of disease symptoms.

This study aimed to investigate the involvement of AratEULS3 in ABA signaling in roots. Our analysis confirms that the transcript levels for ArathEULS3 are upregulated in osmotic stress conditions as well as after exposure to ABA treatment. Promoter::GUS studies suggested that ArathEULS3 is mostly expressed in the roots. Furthermore CRISPR-induced gene knock-out $(\mathrm{KO})$ lines yielded plants with a larger root system and reduced ABA response, suggesting that ArathEULS3 might be important for ABA signaling.

\section{MATERIALS AND METHODS}

\section{Plant Material and Growth Conditions}

Wild type Arabidopsis thaliana seeds, ecotype Columbia 0, were kindly supplied by Prof. Dr. Richard Strasser (Department of Applied Genetics and Cell Biology, University of Natural Resources and Life Sciences, Vienna, Austria). When grown in vitro, seeds were surface sterilized in $70 \%(\mathrm{v} / \mathrm{v})$ ethanol for $2 \mathrm{~min}$, followed by $8 \mathrm{~min}$ in $5 \%$ (v/v) $\mathrm{NaOCl}$ (Sigma-Aldrich, St Louis, MO, USA). Afterwards, the seeds were rinsed eight times with sterile distilled water. The sterilized Arabidopsis seeds were sown in vitro on solid 1/2 Murashige and Skoog (MS) medium (2.154 g/ L MS basal salt [Duchefa Biocheme, Haarlem, The Netherlands], $10 \mathrm{~g} / \mathrm{L}$ sucrose [Duchefa], $0.1 \mathrm{~g} / \mathrm{L}$ myo-inositol [Duchefa], $0.5 \mathrm{~g} / \mathrm{L}$ MES - 2-(N-morpholino)ethanesulfonic acid [Carl Roth, Karlsruhe, Germany], 8 g/L plant tissue culture agar [Duchefa], $\mathrm{pH}$ 5.7) supplemented with $10 \mathrm{mg} / \mathrm{L}$ phosphinothricin (PPT, Duchefa) or $75 \mu \mathrm{g} / \mathrm{ml}$ kanamycin (Duchefa) as selective agents during selection. Alternatively, Arabidopsis seeds were grown in pots containing commercial soil or individually grown in artificial soil (Jiffy-7, $44 \mathrm{~mm} \varnothing$ ). Both plants grown in vitro and in soil were first stratified for 3 days at $4^{\circ} \mathrm{C}$ in the dark and then transferred to a growth chamber at $21^{\circ} \mathrm{C}$ with a $16 / 8 \mathrm{~h}$ light/ dark photoperiod. 


\section{Construction of CRISPR Vectors and Generation of ArathEULS3 Knock-Out Transgenic Lines}

CRISPR vectors (pEN-Chimera and pDe-CAS9) were kindly supplied by Prof. Dr. Holger Puchta (Botanical Institute II, Karlsruhe Institute of Technology, Karlsruhe, Germany). Design of spacer sequences and cloning was performed according to the protocol provided by Schiml et al. (2016). In short, three 20-nucleotide CRISPR spacer sequences targeting three sites within the ArathEULS3 coding sequence (Supplementary File S1, Supplementary Figure S1) were designed using CRISPR-PLANT tool (Xie et al., 2014) and purchased from Invitrogen. In order to obtain entry clones, the 20-nucleotide CRISPR spacer sequences were introduced into pEn-Chimera cut with restriction enzyme BbsI. The customized chimeras were then cloned into pDe-CAS9 by means of Gateway ${ }^{\circledR}$ LR reaction and expression clones were transformed via electroporation to Agrobacterium tumefaciens strain C58C1 pMP90. Arabidopsis thaliana plants, ecotype Col-0 were transformed using the floral dip method (Clough and Bent, 1998).

\section{Evaluation of Germinal Mutation}

Primary transformants (T1) were selected on $1 / 2$ MS medium supplemented with $10 \mathrm{mg} / \mathrm{L}$ PPT. After 7 days in the growth chamber, putative mutants were transferred to artificial soilJiffy- $7^{\circledR}$ for another 11 days. Eighteen-day-old plants were subjected to continuous heat stress for 5 days in a plant cabinet $\left(37^{\circ} \mathrm{C} 16 / 8 \mathrm{~h}\right.$ light/dark photoperiod) and then returned to the growth chamber $\left(22^{\circ} \mathrm{C} 16 / 8 \mathrm{~h}\right.$ light/dark photoperiod) to improve CRISPR efficiency (LeBlanc et al., 2018). The mutations were detected by PCR and sequencing of the fragment flanking the target sequence of Cas9. Progeny (T2) seeds were analyzed by PCR for the presence of the Cas 9 gene and sequencing of the fragment flanking the target sequence. Obtained results were analyzed using BioEdit ${ }^{\circledR}$ software for the presence of double peaks and TIDE (tracking of indels by decomposition) (Brinkman et al., 2014) in order to verify the indel frequencies. A homozygous mutation identified in T2 plants was confirmed in the T3 generation by sequencing. Plants recognized as heterozygous were screened for homozygous mutants in the T3 generation. Two Cas9-free, homozygous $\mathrm{KO}$ lines, one with $1 \mathrm{bp}$ insertion (KO1) and one with 1 bp deletion (KO2) within the ArathEULS3 coding sequence were selected (Procedure described in more detail in Supplementary File 1).

\section{ArathEULS3 OE Lines}

Plants overexpressing ArathEULS3 (lines OE3 and OE4) were generated and screened by Van Hove et al. (2014). The ArathEULS3 transcript levels in two OE lines were determined for 18-day-old in vitro grown seedlings. qRT-PCR analysis revealed approximately 100 -fold increase in transcript levels for both lines (Supplementary Figure S4A). To confirm the presence of the lectin in the $\mathrm{OE}$ lines, crude protein extracts were analyzed by Western Blot analysis, using a polyclonal antibody raised against the EUL domain. Two immunoreactive bands representing the ArathEULS3 protein $(35.6 \mathrm{kDa})$ were clearly detected in protein extracts from plants overexpressing the EUL protein. No signal was detected in extracts from the WT plants, most likely due to the very low lectin content in young plants (Supplementary Figure S4B).

\section{Construction of pArathEULS3:GUS Fusions}

A 2,252 bp promoter fragment including the $5^{\prime}$ UTR region of ArathEULS3 gene was amplified and cloned using the Gateway ${ }^{\circledR}$ Technology (Life Technologies, Carlsbad, CA, USA). In a first PCR the promoter region was amplified using Platinum $P f x$ DNA Polymerase (Life Technologies). In the second PCR the att $B$ sites were added and the PCR product was ligated in the pJET2.1 vector with the CloneJET PCR Cloning kit according to the manufacturer's instructions (Life Technologies). The promoter sequence was confirmed by sequencing (LGC Genomics, Berlin, Germany). Equimolar amounts of the attB flanked PCR product and the donor vector (pDONR221) were used in an overnight $\mathrm{BP}$ recombination reaction with the $\mathrm{BP}$ Clonase $^{\circledR}$ II enzyme mix. The obtained entry clones were subsequently recombined with the destination vector pKGWFS7 to create an expression clone which was transformed via electroporation to Agrobacterium tumefaciens strain $\mathrm{C} 58 \mathrm{C} 1 \mathrm{pMP} 90$. Transgenic plants were made using the floral dip method described above. Transformed seeds were selected on $1 / 2$ MS medium containing $75 \mu \mathrm{g} / \mathrm{ml}$ kanamycin (Duchefa Biocheme, Haarlem, The Netherlands). Two independent, homozygous, transgenic GUS lines were selected, the seeds were multiplied and T4 generation seeds were used in all experiments.

\section{Histochemical Analyses for GUS Staining}

To check the activity of the ArathEULS3 promoter, two homozygous GUS lines were germinated and grown in vertical position on $1 / 2$ MS medium for 0,3 , and 8 days after germination (DAG). Mature siliques, flowers, and fully expanded rosette leaves were detached from 58-day-old-plants. Histochemical staining of $\beta$-glucuronidase (GUS) was performed according to Jefferson et al. (1987). In short, harvested plants were first incubated in $90 \%$ acetone for $30 \mathrm{~min}$ at $4^{\circ} \mathrm{C}$. Subsequently, the plants were washed $3 \times 5 \mathrm{~min}$ in phosphate buffer $(0.1 \mathrm{M}$ $\mathrm{NaH}_{2} \mathrm{PO}_{4} \cdot \mathrm{H}_{2} \mathrm{O}$ [VWR, Darmstadt, Germany] and $0.1 \mathrm{M}$ $\mathrm{Na}_{2} \mathrm{HPO}_{4}$ [VWR], $\mathrm{pH} 7.0$ ) and then incubated at $37^{\circ} \mathrm{C}$ for 30 min in the GUS pre-incubation buffer (phosphate buffer containing $0.5 \mathrm{mM} \mathrm{K}$-ferricyanide and $0.5 \mathrm{mM} \mathrm{K}$ ferrocyanide). Then they were stained in the GUS assay buffer (GUS pre-incubation buffer containing $2 \mathrm{mM}$ 5-bromo-4chloro-3-indolyl $\beta$-D-glucuronic acid [Thermo Scientific, Madison, USA]) and incubated overnight $(18 \mathrm{~h})$ at $37^{\circ} \mathrm{C}$. The reaction was stopped by washing plants in phosphate buffer, followed by incubation in $70 \%$ ethanol for removal of chlorophyll. 


\section{ArathEULS3 Promoter Analyses in Stress Conditions}

Two hormones, $10 \mu \mathrm{M}$ 1-naphthaleneacetic acid (NAA) and 100 $\mu \mathrm{M} \mathrm{ABA}$ and two abiotic stresses, salt $(150 \mathrm{mM} \mathrm{NaCl})$ and drought (20\% w/v PEG6000) were used in stress assays including pArathEULS3::GUS lines and WT plants. $1 \frac{1}{2}$ MS medium was used as a control for salt and drought treatment. Since the stock solutions for the hormones (ABA and NAA) were made in ethanol, control plants were kept on liquid medium containing $0.001 \%$ ethanol. For analyses of ArathEULS3 expression in stress conditions the WT seeds were germinated and grown in vitro on top of a $20 \mu \mathrm{M}$ nylon mesh overlaid on $1 / 2 \mathrm{MS}$ medium. Fourteenday-old seedlings were subjected to stresses by transferring seedlings on the mesh to new Petri dishes filled with $1 / 2$ MS liquid medium containing the indicated concentrations of hormones or PEG6000, and incubated at $21^{\circ} \mathrm{C}$ for 5 or $12 \mathrm{~h}$, collected, and frozen in liquid nitrogen. To assess changes in the expression pattern after stress (ABA, NAA, salt, and drought), 8day-old seedlings of $\mathrm{pArathEULS3::GUS} \mathrm{lines,} \mathrm{grown} \mathrm{vertically}$ on top of a $20 \mu \mathrm{M}$ nylon mesh overlaid on $1 / 2$ MS medium were subjected to stress as described above. For each treatment at least 6 seedlings were collected and the GUS histochemical staining was performed.

\section{Real-Time Quantitative RT-PCR}

Total RNA was extracted using TriReagent ${ }^{\circledR}$ (Sigma-Aldrich). DNase I (Thermo Scientific) was used to digest single- and double stranded DNA. RNA concentration and quality was determined with the NanoDrop 2000 spectrophotometer (Thermo Scientific). First-strand cDNA was synthesized from 1 $\mu \mathrm{g}$ of total RNA with oligo(dT)25 primers and $200 \mathrm{U}$ of M-MLV reverse transcriptase (Thermo Scientific) and then diluted 2.5 times. The quality of the cDNA was tested in a standard reverse transcriptase PCR (RT-PCR) using the reference gene Protein phosphatase 2A (PP2A). PCR amplification products were checked on a $1.5 \%$ agarose gel. Quantitative real-time PCR (qRT-PCR) was performed with the 96-well CFX Connect ${ }^{\mathrm{TM}}$ Real-Time PCR Detection System (Bio-Rad Laboratories, Hercules, CA, USA) using the SensiMix ${ }^{\mathrm{TM}}$ SYBR $^{\circledR}$ No-ROX One-Step kit (Bioline Reagents Limited, London, UK). Reactions were conducted in a total volume of $20 \mu \mathrm{l}$ containing $2 \mu \mathrm{l}$ cDNA template, $1 \times$ SensiMix $^{\text {TM }}$ SYBR $^{\circledR}$ NoROX One-Step mix, and $500 \mathrm{nM}$ gene specific forward and reverse primer. Three independent biological replicates with each two technical replicates were analyzed, each containing a pool of approximately fifty seedlings. qRT-PCR was performed under following conditions: $10 \mathrm{~min}$ at $95^{\circ} \mathrm{C}, 45$ cycles of $15 \mathrm{~s}$ at $95^{\circ} \mathrm{C}, 25$ $\mathrm{s}$ at $60^{\circ} \mathrm{C}$, and $20 \mathrm{~s}$ at $72^{\circ} \mathrm{C}$ and a melting curve was generated after every $\mathrm{qRT}-\mathrm{PCR}$ run. All expression data were normalized using three reference genes: PP2A, TIP41 and UBC9 (Czechowski et al., 2005). All melting curves were analyzed after each run (Bio-Rad CFX Manager 3.1 software) and reference gene stability and quality control of the samples were validated in the qBASEPLUS software (Hellemans et al., 2007). Gene specific primers were evaluated by verification of the amplicon size and sequence as well as determination of the amplification efficiency (Supplementary Table S4).

\section{Root Phenotype in Normal and Stress Conditions}

Arabidopsis thaliana seeds with a gene $\mathrm{KO}$ or overexpressing ArathEULS3, were germinated on square Petri dishes $(120 \times 120$ $\mathrm{mm})$. After 3 days of stratification at $4^{\circ} \mathrm{C}$, Petri dishes were placed in the growth chamber in the upright $\left(78^{\circ}\right.$ angle) position in order to allow the visualization of root growth. To assess whether the stress has an impact on the root growth, 3-day-old seedlings (WT, OE3, OE4, KO1, and $\mathrm{KO} 2$ ) grown in-vitro were transferred in septic conditions to a new $1 / 2 \mathrm{MS}$, solid medium supplemented with $10 \mu \mathrm{M}$ abscisic acid (ABA), salt $(150 \mathrm{mM}$ $\mathrm{NaCl}$ ), or PEG6000 with a water potential of $-0.5 \mathrm{MPa}$ corresponding to a concentration of about $12 \%(\mathrm{w} / \mathrm{v})$. Due to the fact that in the presence of PEG6000 agar used to make plates cannot solidify, the PEG-infusion method described by Van Der Weele et al. (2000) was used. Since the stock solution for ABA was made in ethanol, control plants were kept on $1 / 2$ MS medium containing $0.001 \%$ ethanol. After 10 days in the growth chamber the plates were scanned. The root length and lateral root density of three biological replicates with a minimum of 17 measurements per condition were analyzed from the pictures using the freeware imaging software package Fiji (Schindelin et al., 2012). When grown on medium supplemented with PEG large variations in the number of emerged lateral roots were observed within each genotype. Therefore, a scoring system was developed for the evaluation of lateral root density, based on the number of emerged lateral roots per $\mathrm{cm}$ of root at 7 days post transfer onto a medium with stress: score 0 , no lateral roots; score 1 , lateral root density $0-0.4$ roots $/ \mathrm{cm}$; score 2 , lateral root density $0.41-0.8$ roots $/ \mathrm{cm}$; score 3 , lateral root density $0.81-1.2$ roots $/ \mathrm{cm}$; score 4 , lateral root density $>1.2$ roots $/ \mathrm{cm}$.

\section{Statistical Analysis}

Statistical analysis was conducted using SPSS Statistics 22 (IBM) and the data were considered statistically significant for $\mathrm{p}<0.05$. The assumption of normality was tested with the ShapiroWilkinson test and the equality of variances of normally distributed data was assessed using the Levene's test. Comparisons between the WT and the transgenic lines were made by using Independent-samples T-test with Bonferroni correction for normally distributed data or Mann-Whitney $U$ test for not normally distributed data. All results for normally distributed data are shown as means \pm SE (standard error) or SD (standard deviation) or median values when data were not normally distributed. Statistical analysis for lateral root scoring was performed using the Jonckheere-Terpstra test, which is better suited for ordinal data $\left(\mathrm{p}<0.05\right.$, ${ }^{* *} \mathrm{p}<0.01$, ${ }^{* *} \mathrm{p}<$ $0.001)$. Statistical analysis for qRT-PCR data was evaluated with the REST-384 software using the pair wise fixed reallocation randomization test (with 2,000 randomizations) (Pfaffl et al., 2002). 


\section{RESULTS}

\section{GUS-Reporter Gene Analysis of ArathEULS3 Promoter}

To determine the tissue specific ArathEULS3 expression, promoter::GUS studies were performed. A construct comprising 2,252 bp upstream of the translation start was fused to a reporter gene encoding $\beta$-glucuronidase (GUS). Homozygous transgenic lines were selected and analyzed for promoter activity throughout plant development using histochemical staining. No expression was observed in very young, 1-day-old seedlings (Figure 1A) even after 18 h GUS staining. Promoter activity was observed in the root cap starting from 3 days after germination (DAG) and in the root-hypocotyl transition zone, particularly in the cells flanking the adventitious root primordium (Figures 1B-D). Analysis of plantlets at 8 DAG yielded GUS staining only in the roots (Figure 1E); mainly in the oldest parts of the root (Figures 1F-M). The expression was detected in the central root cylinder in mature parts of the root differentiation zone (Figures 1F, G) as well as in cells flanking young lateral root primordia (Figure 1J). Patchy staining was observed in the younger part of the root differentiation zone (Figures $\mathbf{1} \mathbf{K}-\mathbf{M}$ ). No expression was present in the root meristem, endodermis, cortex, and epidermis. Staining experiments of the aerial plant parts for different plant developmental stages revealed no ArathEULS3 expression in the leaf tissues (data not shown).

\section{Stress Regulates the Expression Level and Pattern for ArathEULS3}

Expression of ArathEULS3 was examined in 14-day-old seedlings subjected to ABA, auxin (NAA), and osmotic (20\% PEG) stress for 5 and $12 \mathrm{~h}$. Transcript levels for ArathEULS3 were determined by qRT-PCR analysis (Figure 2A). COR15A gene was used as a control for ABA treatment (Chen et al., 2009). The qRT-PCR analysis revealed a significant upregulation of COR15A in both ABA and PEG treatment (Supplementary Figure S3), which indicates that plants sensed the stress treatments. The expression level of ArathEULS3 was not affected by auxin treatment but both $\mathrm{ABA}$ and osmotic stress caused a significant increase in the transcript levels. The strongest effect on ArathEULS3 expression was observed after $5 \mathrm{~h}$ of ABA treatment (13-fold upregulation after $5 \mathrm{~h}$ treatment and 5 -fold after $12 \mathrm{~h}$ treatment). Osmotic stress induced by PEG caused a small, but significant upregulation of about 3 fold both after 5 and $12 \mathrm{~h}$.

To determine the promoter activity in stress conditions 8day-old pArathEULS3::GUS lines were subjected to ABA, NAA, salt, and drought stress for 5 and $12 \mathrm{~h}$. Overnight GUS staining after $\mathrm{ABA}$ treatment revealed increased promoter activity in the root tips of more than $90 \%$ of the plants tested (Figure $\mathbf{2 B}$ ). The staining in the root tips was stronger in $70 \%$ of the plants subjected to $5 \mathrm{~h}$ PEG treatment and $35 \%$ of the plants after 12 h PEG. No clear differences in staining patterns were observed for plants treated with auxin or salt.

\section{ArathEULS3 KO Mutants Show a Reduced Inhibition of Primary Root Growth and Lateral Root Formation in Normal and Osmotic Stress Conditions}

To investigate the role of ArathEULS3 in plant responses to salt and osmotic stress the root growth phenotype of two KO lines and two OE lines was examined in the presence of salt $(150 \mathrm{mM}$ $\mathrm{NaCl})$, drought (PEG), and $\mathrm{ABA}(10 \mu \mathrm{M})$. The construction and selection of ArathEULS3 KO lines is described in detail in the Supplementary File S1. The growth of ArathEULS3 KO and overexpression lines was analyzed in non-stressed conditions (mock treatment). The roots of both KO lines were significantly longer than the roots of WT plants, whereas the root length of one of the $\mathrm{OE}$ lines was significantly shorter (Figure 3). The lateral root density did not differ in any of the mutant lines. No significant differences were observed in different mock treatments (Supplementary Figures S5 and S6).

After treatment with PEG and salt both KO lines revealed a longer primary root compared to WT plants (Figures 4A, B, D, E). However, after treatment with $\mathrm{ABA}$ only the roots of $\mathrm{KO} 2$ were significantly longer compared to roots of WT plants (Figures 5A, B). When grown in osmotic (PEG) stress conditions both $\mathrm{KO}$ mutants had a substantially higher number of emerged lateral roots compared to WT plants
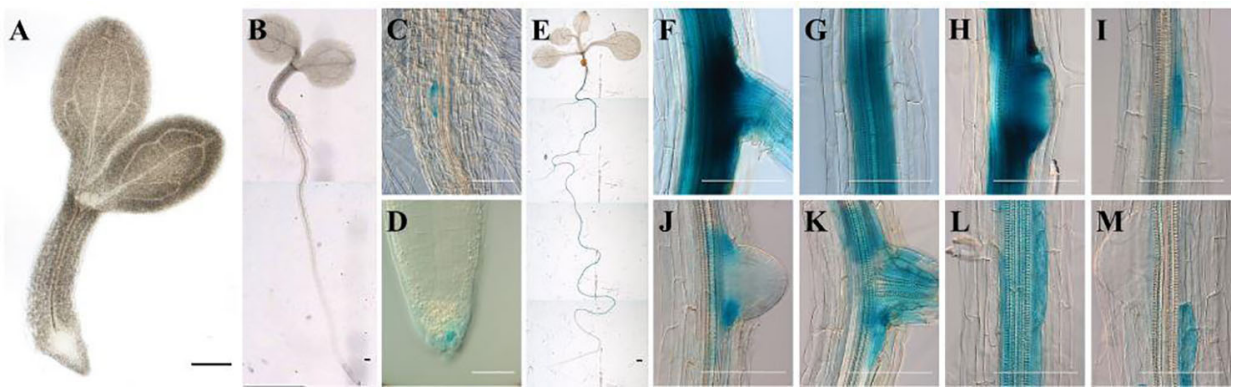

FIGURE 1 | Expression pattern of pArathEULS3::GUS in Arabidopsis thaliana seedlings. (A) 1-day-old seedlings show no expression, (B-D) GUS staining in the root-hypocotyl transition zone and root cap of 3-day-old seedling, (E-M) high expression in the roots of 8-day-old plants. Panels F-M show successive selected parts moving down the same root. GUS staining was carried out for $18 \mathrm{~h}$. Scale bars represent $0.1 \mathrm{~mm}$ for images C, D and E-M, $1 \mathrm{~mm}$ for A, B, E. 


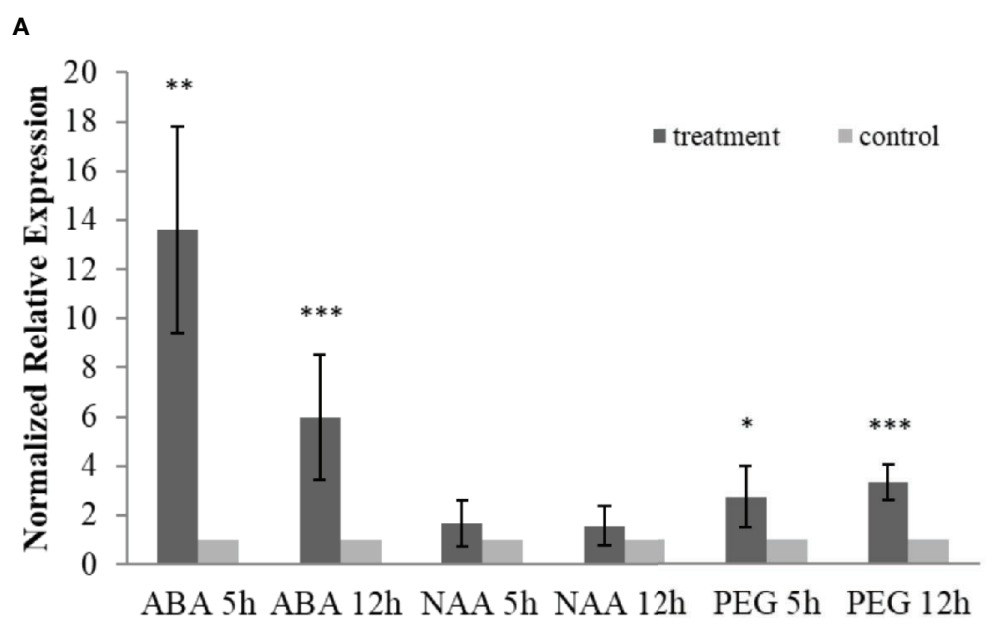

B

Control $100 \mu \mathrm{M}$ ABA Control PEG

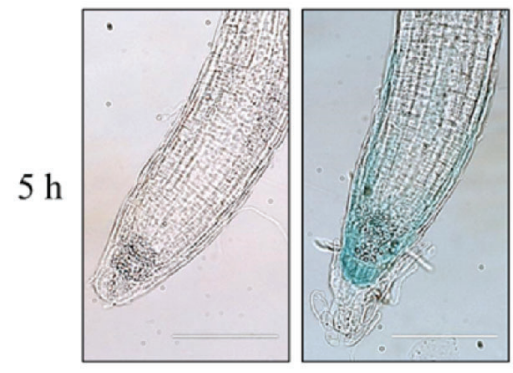

$12 \mathrm{~h}$
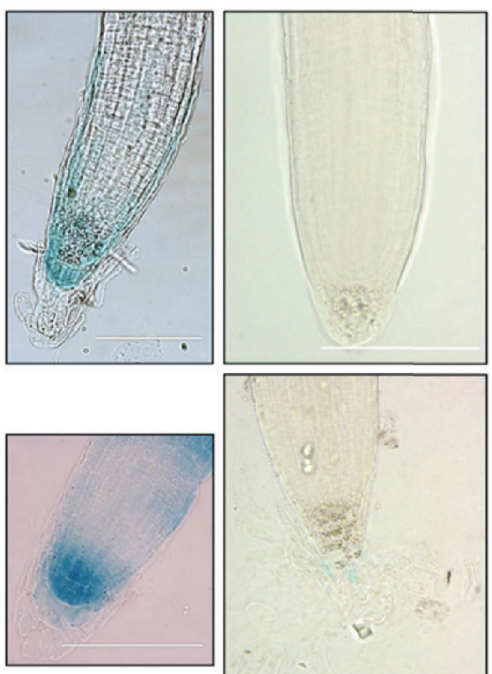

$20 \%$ PEG
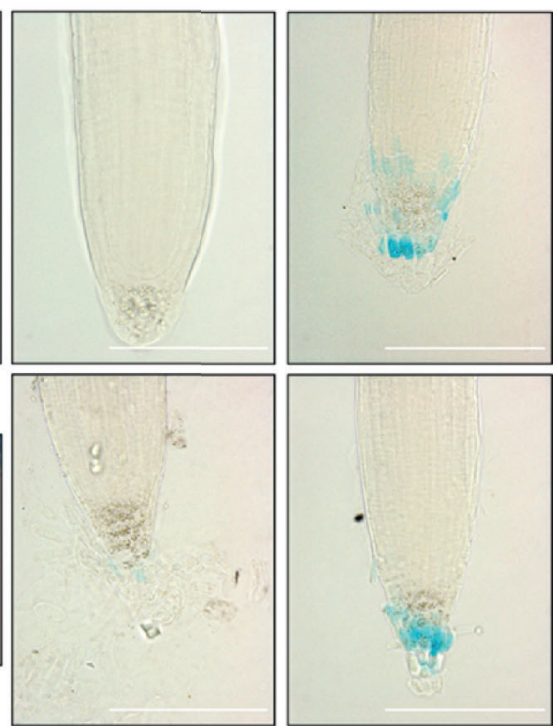

FIGURE 2 | Stress responsiveness of ArathEULS3 expression. (A) Normalized relative expression of ArathEULS3 in 14-day-old seedlings after ABA, NAA, and PEG treatment. Bars represent means \pm SE normalized relative ArathEULS3 expression compared to mock-treated plants. Asterisks indicate statistically significant differences to the expression level in mock treated seedlings ( ${ }^{*} \mathrm{p} \leq 0.05,{ }^{* \star} \mathrm{p} \leq 0.01,{ }^{\star \star \star} \mathrm{p} \leq 0.001$; REST analysis). The graphs represent the results of three independent biological replicates. (B) GUS staining of 8-day-old pArathEULS3::GUS lines treated with ABA and PEG for 5 and 12 h, compared to mock-treated plants. GUS staining was performed for $18 \mathrm{~h}$. GUS staining was observed in more than $90 \%$ of the plants treated with ABA. GUS staining was apparent in $70 \%$ and $35 \%$ of the plants after 5 and $12 \mathrm{~h}$ of PEG treatment, respectively. The scale bar represents $0.1 \mathrm{~mm}$.

(Figure 4C). Fewer differences were observed in lateral root density for plants grown on high salinity medium (Figure 4F). Since ABA is known to mediate the inhibition of lateral root emergence in osmotic stress conditions (Deak and Malamy, 2005), the changes in lateral root number and the lateral root density were examined after exposure to $10 \mu \mathrm{M}$ ABA concentrations. Neither the lateral root number nor the lateral root density in the transgenic lines differed significantly from the WT (Figures 5C, D). Taken together, these results suggest that ArathEULS3 KO lines revealed a root phenotype which is characterized by a faster root growth. Roots of $\mathrm{KO}$ lines are less sensitive to osmotic stress compared to WT plants.

Since the root length of ArathEULS3 KO lines is larger than the root length of the WT plants under both unstressed and stressed conditions, the differences in root length between mock treatments and different stress conditions, including ABA, PEG, and salt treatments, were quantified. This analysis revealed that compared to non-stress conditions the average difference in root lengths between $\mathrm{KO}$ and WT plants grown in the presence of stress was considerably larger, irrespective of the stress factor used (Table 1). 

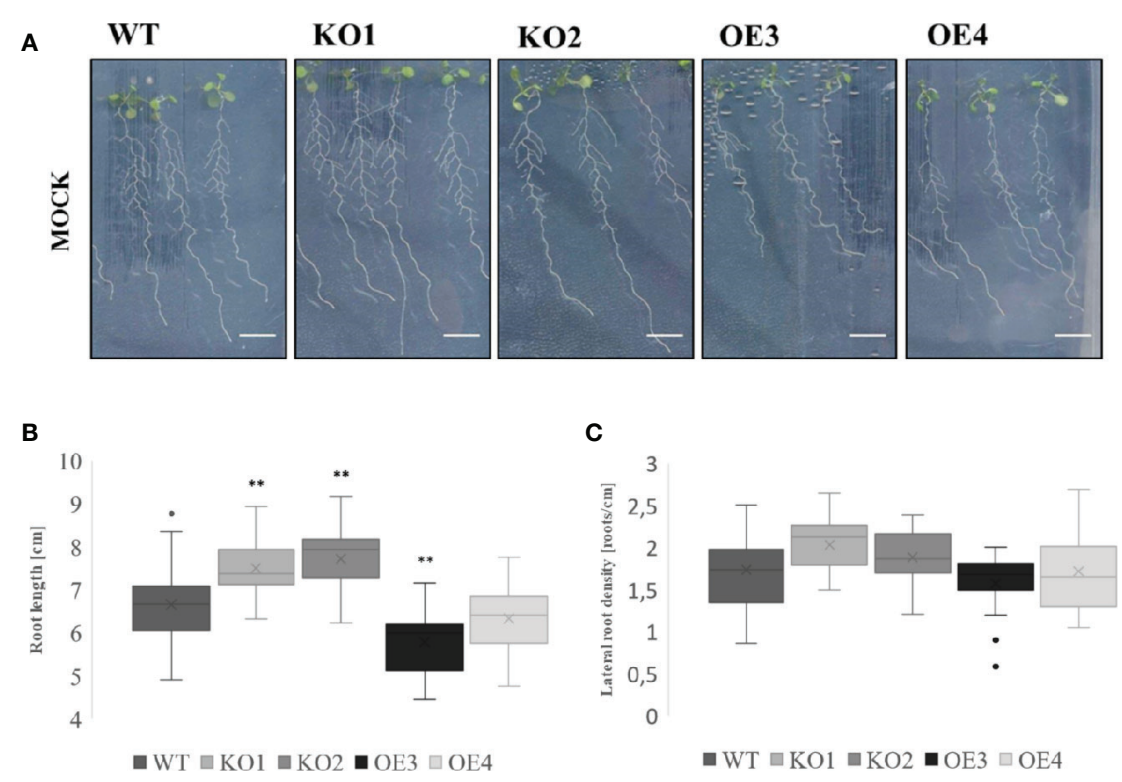

FIGURE 3 | Growth of transgenic lines under normal growth conditions. (A) Root phenotype, (B) root length, (C) and lateral root density of 10 day-old transgenic lines compared to WT. The graphs represent three biological replicates with minimum 17 data points per condition. The comparison between the root length of WT and transgenic lines was made by using Independent-samples T-test for normally distributed data. The comparison of lateral root density of different transgenic lines to WT was made by using Mann-Whitney test for not normally distributed data. The distributions of root length and lateral root density values are shown as box and whisker plots. The boxes represent the $25-75$ th percentiles, the median is indicated as a horizontal line, the mean is indicated as a cross, the outliers are represented as circles. The whiskers are equal to $1.5 \times$ the interquartile range $\left({ }^{*} p<0.05,{ }^{\star *} p<0.01\right)$.

\section{DISCUSSION}

ABA is an important phytohormone which in non-stress conditions is maintained in the plant at low levels. The basal ABA concentration is important for primary plant metabolism, supports plant growth and development, and has an inhibitory effect on leaf emergence and promotes root growth (Yoshida et al., 2019). PEG is a high molecular weight solute which can mimic drought stress (Alqurashi et al., 2018) and ABA is one of the most important transducer of drought, salt, and osmotic stresses (Harris, 2015). Our results suggest that exposure of WT plants to ABA and PEG results in an increased mRNA level for ArathEULS3 in the WT plants. Rasheed et al. (2016) also reported ArathEUSL3 as one of the highly up-regulated genes during progressive drought stress. The mRNA levels were 4-fold upregulated in the roots already at 3 days after ceasing the watering and increased after further exposure to drought stress to 5.5-, 8.1-, and 12.6-fold up-regulation after 3, 5, and 7 days, respectively. Interestingly the significant upregulation of ArathEULS3 mRNA levels in the shoots was only observed after 7 and 9 days (4- and 7.3-fold, respectively). Additionally the gene expression analysis after osmotic stress $(300 \mathrm{mM}$ mannitol) reported by Kilian et al. (2007) revealed changes in ArathEULS3 mRNA levels both in the roots (2.8-fold after $1 \mathrm{~h}$ of treatment, up to 8.8 -fold after $24 \mathrm{~h}$ ) and in the shoots (5.5-fold after $3 \mathrm{~h}$, up to 22 -fold after $24 \mathrm{~h}$ ). Similar, but slightly milder effects were observed after salt treatment $(150 \mathrm{mM} \mathrm{NaCl})$, with a maximum of 5.4-fold upregulation in the roots after $6 \mathrm{~h}$ and in the shoots after $24 \mathrm{~h}$ of treatment (13.12-fold upregulation). Moreover, several other recent publications linked ArathEULS3 to ABA signaling. ArathEULS3 was shown to interact with CPK3 (Figure 6), a calcium-dependent protein kinase (Berendzen et al., 2012) which has an important function in the guard cells and ABA signaling. Pandey et al. (2010) recognized CPK3 as an ABAregulated gene with 6 -fold upregulation in guard cells and leaves after $3 \mathrm{~h}$ of $50 \mu \mathrm{M}$ ABA treatment on 5-week-old Arabidopsis leaves. Similarly, AratEULS3 mRNA levels were 5.6-fold upregulated in Arabidopsis cell suspension cultures after $20 \mathrm{~h}$ of $50 \mu \mathrm{M}$ ABA treatment (Böhmer and Schroeder, 2011). Furthermore, Wang et al. (2011) listed ArathEULS3 among the 67 most important ABA-induced genes, specifically in stomata. Arabidopsis overexpressing ArathEULS3 showed fewer disease symptoms after infection with $P$. syringae (Van Hove et al., 2015) and revealed increased tolerance to drought stress ( $\mathrm{Li}$ et al., 2014). Additionally, the in-silico promoter analysis confirmed the presence of many cis-regulatory elements associated with drought/salt and $\mathrm{ABA}$ response in the promoter region upstream from the ArathEULS3 gene (Van Hove et al., 2014).

Our promoter activity analysis with the GUS reporter system did not reveal any staining in leaf tissues for any of the tested developmental stages, not even after any of the stress treatments. GUS staining was mostly detected in the roots; especially in the central cylinder of the differentiation zone and in the cells flanking young lateral root primordia. However, the identification of the cell wall proteome revealed the presence of EUL peptides in A. thaliana leaves (Hervé et al., 2016). The lack of GUS expression in leaves in 


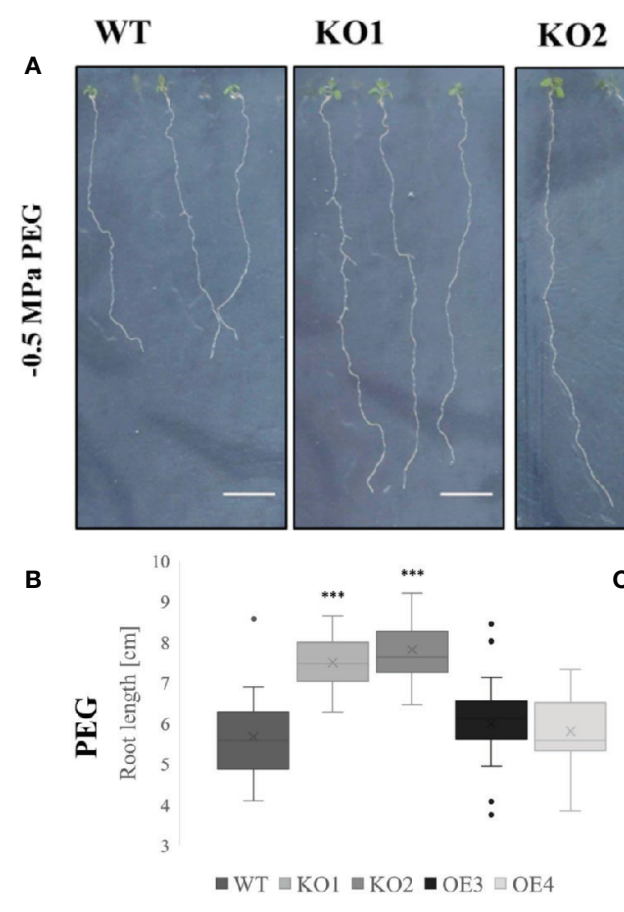

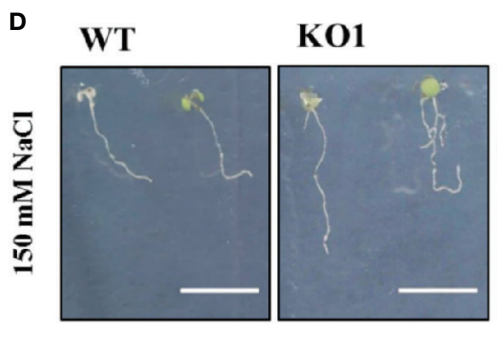

$\mathrm{KO} 2$

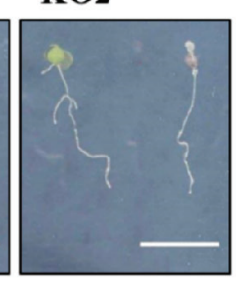

OE3

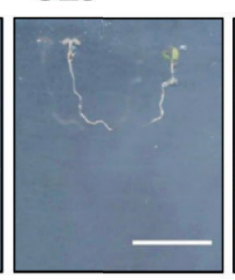

OE4

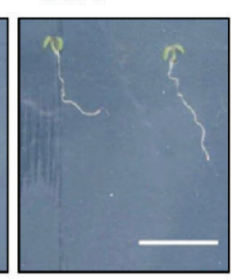

$\mathbf{E}$
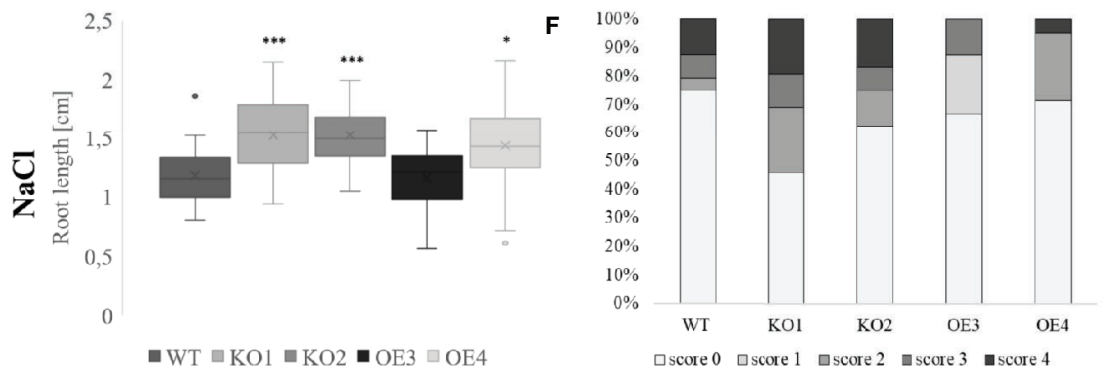

OE3

OE4

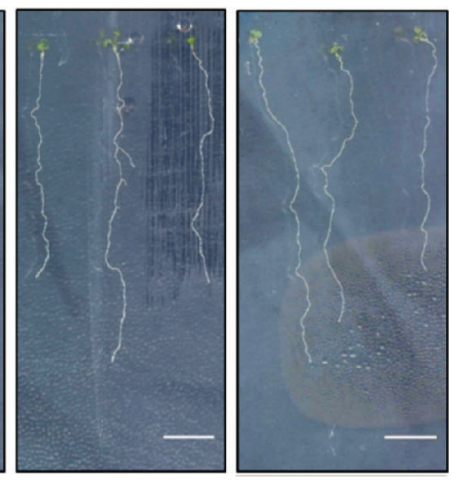

$100 \%$
$90 \%$
$80 \%$
$70 \%$
$60 \%$
$50 \%$
$40 \%$
$30 \%$
$20 \%$
$10 \%$
$0 \%$

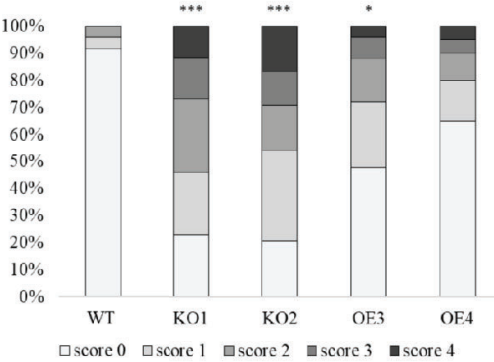

FIGURE 4 | Growth of transgenic lines after salt and osmotic stress treatments. (A, D) Root phenotype, (B, E) root length and (C, F) lateral root density of WT and transgenic lines grown on medium with or without PEG (-0.5 MPa) or salt (150 mM). The graphs represent three biological replicates with minimum 17 data points per condition. Scoring system for evaluation of lateral root density is explained in the Materials and Methods. The seeds of WT plants and transgenic lines (KO1, $\mathrm{KO} 2, \mathrm{OE} 3$, and OE4) were germinated in vitro for 3 days and the young seedlings were then transferred onto a medium containing stress factors (150 mM NaCl or -0.5 MPa PEG6000) for another 7 days. The comparison between the root length of WT and transgenic lines was made by using Independent-samples T-test for normally distributed data. The distributions of root length values are shown as box and whisker plots. The boxes represent the 25-75th percentiles, the median is indicated as a horizontal line, the mean is indicated as a cross, the outliers are represented as circles. The whiskers are equal to $1.5 \times$ the interquartile range. The comparison between the scoring of lateral root density between WT and transgenic lines was made by using Jonckheere-Terpstra test. ( ${ }^{\star} p<0.05$, ${ }^{\star \star \star} p<0.001$ ).

our experiments might be explained by the promoter region used for the analysis. It is well-established that transcription regulatory elements are present not only in the promoter sequence preceding the gene sequence but are also located within intron sequences or distal enhancers which can be located even thousands of nucleotides up- or downstream from the coding sequence (Le Hir et al., 2003; Bulger and Groudine, 2011). The absence of such regulatory sequences might explain the lack of promoter activity in the leaves in our experiment, though transcripts for ArathEULS3 have been detected multiple times before in leaves collected at different 


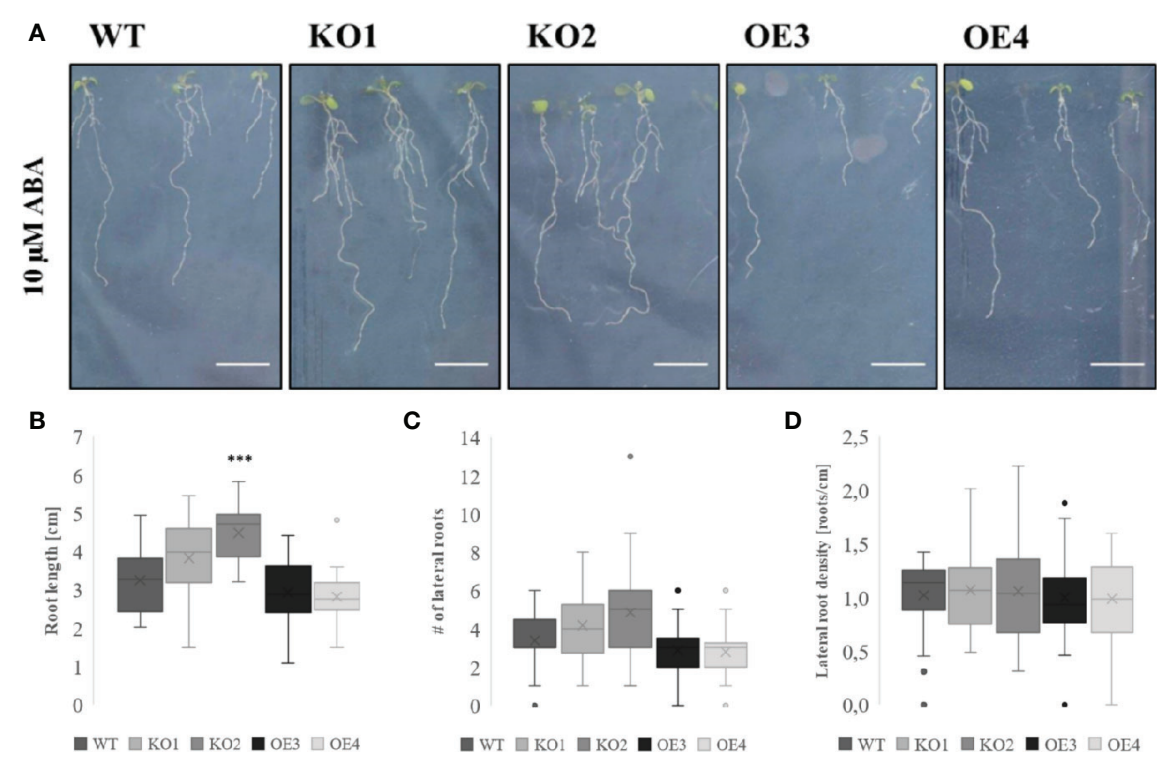

FIGURE 5 | Growth of transgenic lines after ABA treatment. (A) Root phenotype and (B) root length of WT and transgenic lines grown in the presence or absence of $10 \mu \mathrm{M}$ ABA. (C) Number of lateral roots and (D) lateral root density in plants grown on medium supplemented with ABA. The graphs represent three biological replicates with minimum 17 data points per condition. The seeds of WT plants and transgenic lines (KO1, KO2, OE3, and OE4) were germinated in vitro for 3 days and the seedlings were transferred onto a medium containing $10 \mu \mathrm{M}$ ABA for another 7 days. The comparison between the root length of WT and transgenic lines was made by using Independent-samples T-test for normally distributed data. The statistical analysis for the number of lateral roots and lateral root density was performed using Mann-Whitney $U$ test for not normally distributed data. The distributions of root length, number of lateral roots, and lateral root density values are shown as box and whisker plots. The boxes represent the $25-75$ th percentiles, the median is indicated as a horizontal line, the mean is indicated as a cross, the outliers are represented as circles. The whiskers are equal to $1.5 \times$ the interquartile range $\left({ }^{\star \star} \mathrm{p}<0.001\right)$.

TABLE 1 | Differences in root length for WT plants and KO lines grown in the presence or absence of a stress factor.

\begin{tabular}{lcc}
\hline & \multicolumn{2}{c}{ Root length difference } \\
\cline { 2 - 3 } & KO1 compared to WT & KO2 compared to WT \\
\hline MOCK $(+0.001 \% \mathrm{EtOH})$ & $8.5 \%$ & $13.3 \%$ \\
$10 \mu \mathrm{M} \mathrm{ABA}$ & $17.7 \%$ & $38.0 \%$ \\
$\mathrm{MOCK}$ & $12.7 \%$ & $16.0 \%$ \\
$-0.5 \mathrm{MPa} \mathrm{PEG}$ & $32.3 \%$ & $37.9 \%$ \\
$150 \mathrm{mM} \mathrm{NaCl}$ & $28.9 \%$ & $28.9 \%$ \\
\hline
\end{tabular}

developmental stages (Wang et al., 2011; Van Hove et al., 2014). After exposure of plants expressing pArathEULS3::GUS to ABA an increase of GUS expression in the root tips, more specifically in the root cap, was apparent, which is reminiscent of the expression pattern obtained using an abscisic acid responsive element (ABRE)based synthetic promoter (Fujita et al., 2013). Together with the evidence from previous studies (Kilian et al., 2007; Böhmer and Schroeder, 2011; Wang et al., 2011; Van Hove et al., 2014; Rasheed et al., 2016) our results indicate that ArathEULS3 is definitely an ABA-responsive gene.

Since ArathEULS3 is the only representative of the EUL family in the model plant Arabidopsis thaliana, functional redundancy is excluded. This makes Arabidopsis a good candidate for studying the physiological importance of EUL proteins in plants. In our analyses we used two independent, homozygous OE lines and two homozygous KO lines. The use of two mutant lines may be a shortcoming of this study, since it was not always sufficient to draw straight forward conclusions. Our root phenotype analysis revealed that the root architecture of CRISPR ArathEULS3 KO lines differed significantly from the WT plants. Both KO lines yielded a larger root system in normal growth conditions, and one of the ArathEULS3 overexpression lines had significantly shorter roots. ABA regulates the primary and lateral root growth in response to drought or high salinity. Low concentrations of ABA promote the root growth whereas high concentrations inhibit both the primary and the lateral root growth by inhibiting cell division in the meristem (Harris, 2015). Plants defective in ABA biosynthesis are characterized by a larger root system and impaired inhibition of lateral root elongation. ArathEULS3 was reported to interact with the ABA receptor PYL9 (Figure 6), one of the most important players in ABA signaling (Li et al., 2014). Similar to ArathEULS3, the expression of PYL9 was most dominant in the vascular root tissues (Antoni et al., 2013). Together with PYL8, PYL9 is the most strongly induced gene of this family after ABA treatment (Tischer et al., 2017), and both PYL8 and PYL9 were shown to be important for regulating root architecture (Xing et al., 2016). Interestingly, when overexpressing the PYL9 gene the authors observed a reduced number of lateral roots and a shorter primary root. The double mutant $p y l 8-p y l 9$ revealed a longer root when grown on medium supplemented with $\mathrm{ABA}$ resulting in a reduced 


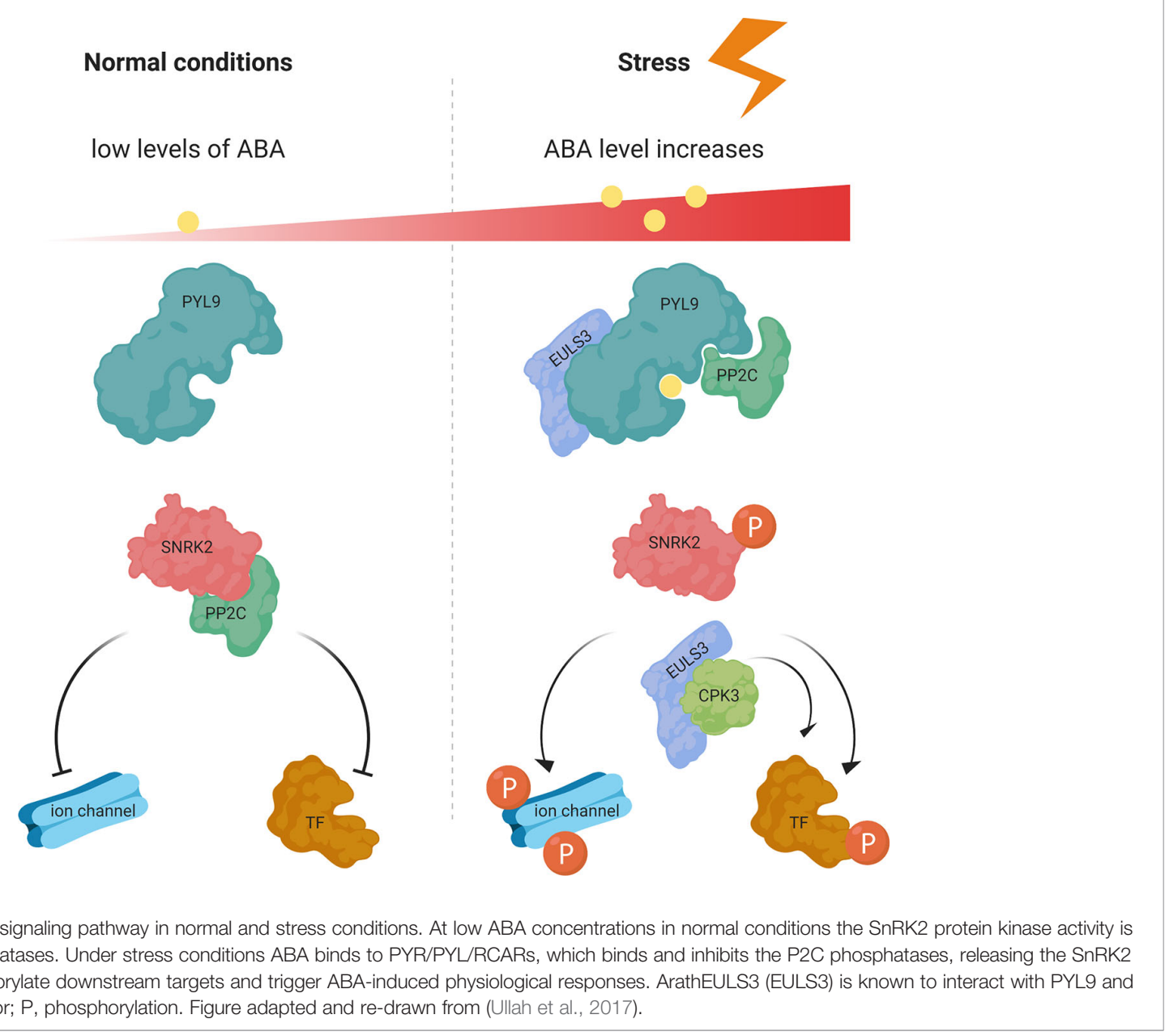

sensitivity to ABA-mediated primary root growth inhibition and a slightly increased number of lateral roots (Xing et al., 2016), suggesting that PYL8 and PYL9 mediate the ability of ABA to inhibit primary root growth. In our analyses, only the ArathEULS3 KO2 line showed a significant increase in the root length after $\mathrm{ABA}$ treatment. However the root length of both KO lines was increased in osmotic stress conditions (salt, PEG) compared to WT plants. These data together with the results showing the increase of ArathEULS3 expression in the root tips upon ABA treatment suggest that ArathEULS3 could be important for the inhibition of cell division or cell elongation controlled by the ABA-signaling in the root cap in osmotic stress conditions.

Auxin plays a pivotal role in the coordination of lateral root primordial formation and lateral root growth (Vilches-Barro and Maizel, 2015). Interestingly, PYL9, was shown to be a node between the $\mathrm{ABA}$ and auxin crosstalk in the roots, through direct interaction with MYB77 and MYB44 transcription factors, regulating their transcriptional activity (Xing et al., 2016). However, ArathEULS3 expression was not influenced by the presence of high auxin concentrations in the environment. The analysis presented here was performed using whole seedlings. Therefore if the response was specific for one tissue type, it could have been overlooked. In that respect, the analysis done by Bargmann et al. (2013) where gene expression was measured locally in the xylem pole pericycle cells, stele, columella, epidermis, and lateral root cap, showed significant downregulation of ArathEULS3 after $3 \mathrm{~h}$ of auxin $(5 \mu \mathrm{M}$ IAA) treatment specifically in the xylem pole pericycle cells as well as in the stele, endodermis, and the lateral root cap. This is in agreement with the hypothesis suggesting that ArathEULS3, like PYL9, could play a role in the interplay of $\mathrm{ABA}$ and auxin signaling in roots.

PYL9 OE lines were characterized by a reduced number of lateral roots (Xing et al., 2016). None of the tested transgenic lines differed in the lateral root number and density from the WT roots under normal growth conditions. It is known that high concentrations of PEG and salt inhibit lateral root emergence. However, our data show that after PEG and salt treatment some of the KO plants clearly had a higher number of emerged lateral roots. Therefore the lateral root density was scored in each plant, which revealed that after transfer to PEG, both KO mutants had a substantially higher number of emerged lateral roots than WT plants. Differences were also observed after salt treatment, however, not statistically significant. Together with specific expression of ArathEULS3 in the cells flanking lateral root 
primordia, these data suggest that ArathEULS3 might play a role in lateral root emergence during stress conditions like water deficit or high salinity. However, this should be confirmed by performing more detailed analyses of cell division in the lateral root primordia and emergence of lateral roots in transgenic lines of ArathEULS3.

Here we present a new set of data suggesting a strong link between ArathEULS3 and ABA responses in roots. The promoter activity assays suggest that ArathEULS3 expression mainly takes place in the root tissues. Furthermore, the KO lines revealed a root phenotype similar to the double $p y l 8$ - $p y l 9$ mutant, a $\mathrm{KO}$ line for the major $\mathrm{ABA}$ receptors after osmotic stress and ABA treatment.

\section{DATA AVAILABILITY STATEMENT}

All datasets generated for this study are included in the article/ Supplementary Material.

\section{AUTHOR CONTRIBUTIONS}

MD, TB, and ED outlined and designed the study. MD performed the experiments. MD analyzed and interpreted the data. MD and ED prepared the manuscript. ED, TB, and GS conceived and

\section{REFERENCES}

Alqurashi, M., Chiapello, M., Bianchet, C., Paolocci, F., Lilley, K. S., and Gehring, C. (2018). Early responses to severe drought stress in the Arabidopsis thaliana cell suspension culture proteome. Proteomes 6, 38. doi: 10.3390/proteomes6040038

Antoni, R., Gonzalez-Guzman, M., Rodriguez, L., Peirats-Llobet, M., Pizzio, G. A., Fernandez, M. A., et al. (2013). PYRABACTIN RESISTANCE1-LIKE8 plays an important role for the regulation of abscisic acid signaling in root. Plant Physiol. 161, 931-941. doi: 10.1104/pp.112.208678

Böhmer, M., and Schroeder, J. I. (2011). Quantitative transcriptomic analysis of abscisic acid-induced and reactive oxygen species-dependent expression changes and proteomic profiling in Arabidopsis suspension cells. Plant J. 67, 105-118. doi: 10.1111/j.1365-313X.2011.04579.x

Banda, J., Bellande, K., von Wangenheim, D., Goh, T., Guyomarc'h, S., Laplaze, L., et al. (2019). Lateral root formation in Arabidopsis: a well-ordered LRexit. Trends Plant Sci. 24, 1-14. doi: 10.1016/j.tplants.2019.06.015

Bao, Y., Aggarwal, P., Robbins, N. E., Sturrock, C. J., Thompson, M. C., Tan, H. Q., et al. (2014). Plant roots use a patterning mechanism to position lateral root branches toward available water. Proc. Natl. Acad. Sci. U. S. A. 111, 9319-9324. doi: 10.1073/pnas.1400966111

Barberon, M., Vermeer, J. E. M., De Bellis, D., Wang, P., Naseer, S., Andersen, T. G., et al. (2016). Adaptation of root function by nutrient-induced plasticity of endodermal differentiation. Cell 164, 447-459. doi: 10.1016/j.cell.2015.12.021

Barberon, M. (2017). The endodermis as a checkpoint for nutrients. New Phytol. 213, 1604-1610. doi: 10.1111/nph.14140

Bargmann, B. O. R., Vanneste, S., Krouk, G., Nawy, T., Efroni, I., Shani, E., et al. (2013). A map of cell type-specific auxin responses. Mol. Syst. Biol. 9, 1-13. doi: $10.1038 / \mathrm{msb} .2013 .40$

Berendzen, K. W., Böhmer, M., Wallmeroth, N., Peter, S., Vesić, M., Zhou, Y., et al. (2012). Screening for in planta protein-protein interactions combining bimolecular fluorescence complementation with flow cytometry. Plant Methods 8, 1-17. doi: 10.1186/1746-4811-8-25 supervised the experiments and critically revised the manuscript. All authors have read, revised, and approved the final manuscript.

\section{FUNDING}

This research was funded by the Research Council of Ghent University (project BOF15/GOA/005).

\section{ACKNOWLEDGMENTS}

We wish to thank Prof. Holger Puchta (Botanical Institute II, Karlsruhe Institute of Technology, Karlsruhe, Germany) for providing CRISPR vectors and Gernot Beihammer (Department of Applied Genetics and Cell Biology, University of Natural Resources and Life Sciences, Vienna, Austria) for his assistance in preparation of the root experiments.

\section{SUPPLEMENTARY MATERIAL}

The Supplementary Material for this article can be found online at: https://www.frontiersin.org/articles/10.3389/fpls.2020.00437/ full\#supplementary-material

Brinkman, E. K., Chen, T., Amendola, M., and van Steensel, B. (2014). Easy quantitative assessment of genome editing by sequence trace decomposition. Nucleic Acids Res. 42, e168. doi: 10.1093/nar/gku936

Bulger, M., and Groudine, M. (2011). Functional and mechanistic diversity of distal transcription enhancers. Cell 144, 327-339. doi: 10.1016/j.cell.2011.01.024

Chen, C. C., Liang, C. S., Kao, A. L., and Yang, C. C. (2009). HHP1 is involved in osmotic stress sensitivity in Arabidopsis. J. Exp. Bot. 60, 1589-1604 doi: 10.1093/jxb/erp039

Clough, S. J., and Bent, A. F. (1998). Floral dip: A simplified method for Agrobacterium-mediated transformation of Arabidopsis thaliana. Plant J. 16, 735-743. doi: 10.1046/j.1365-313X.1998.00343.x

Czechowski, T., Stitt, M., Altmann, T., Udvardi, M. K., and Scheible, W. (2005). Genome-wide identification and testing of superior reference genes for transcript normalization in Arabidopsis thaliana. Scanning 139, 5-17. doi: $10.1104 /$ pp.105.063743.1

Deak, K. I., and Malamy, J. (2005). Osmotic regulation of root system architecture. Plant J. 43, 17-28. doi: 10.1111/j.1365-313X.2005.02425.x

Fouquaert, E., Peumans, W. J., Vandekerckhove, T. T. M., Ongenaert, M., and Van Damme, E. J. M. (2009). Proteins with an Euonymus lectin-like domain are ubiquitous in Embryophyta. BMC Plant Biol. 9, 136. doi: 10.1186/1471-2229-9-136

Fujita, Y., Yoshida, T., and Yamaguchi-Shinozaki, K. (2013). Pivotal role of the AREB/ ABF-SnRK2 pathway in ABRE-mediated transcription in response to osmotic stress in plants. Physiol. Plant 147, 15-27. doi: 10.1111/j.1399-3054.2012.01635.x

Harris, J. (2015). Abscisic acid: hidden architect of root system structure. Plants 4, 548-572. doi: 10.3390/plants4030548

Hellemans, J., Mortier, G., De Paepe, A., Speleman, F., and Vandesompele, J. (2007). qBase relative quantification framework and software for management and automated analysis of real-time quantitative PCR data. Genome Biol. 8, R19. doi: 10.1186/gb-2007-8-2-r19

Hervé, V., Duruflé, H., San Clemente, H., Albenne, C., Balliau, T., Zivy, M., et al. (2016). An enlarged cell wall proteome of Arabidopsis thaliana rosettes. Proteomics 16, 3183-3187. doi: 10.1002/pmic.201600290 
Jefferson, R. A. T., A.Kavanagh', O., and W.Bevan, M. (1987). GUS fusions: Bglucuronidase as a sensitive and versatile gene fusion marker in higher plants. EMBO J. 6, 3901-3907. doi: 10.1002/j.1460-2075.1987.tb02730.x

Kilian, J., Whitehead, D., Horak, J., Wanke, D., Weinl, S., Batistic, O., et al. (2007). The AtGenExpress global stress expression data set: Protocols, evaluation and model data analysis of UV-B light, drought and cold stress responses. Plant J. 50, 347-363. doi: 10.1111/j.1365-313X.2007.03052.x

Le Hir, H., Nott, A., and Moore, M. J. (2003). How introns influence and enhance eukaryotic gene expression. Trends Biochem. Sci. 28, 215-220. doi: 10.1016/ S0968-0004(03)00052-5

LeBlanc, C., Zhang, F., Mendez, J., Lozano, Y., Chatpar, K., Irish, V. F., et al. (2018). Increased efficiency of targeted mutagenesis by CRISPR/Cas9 in plants using heat stress. Plant J. 93, 377-386. doi: 10.1111/tpj.13782

Li, D., Wang, X., Yuan, D., Zhang, L., Jiang, X., Tao, Z., et al. (2014). Overexpression of ArathEULS3 confers ABA sensitivity and drought tolerance in Arabidopsis. Plant Cell. Tissue Organ Cult. 117, 431-442. doi: 10.1007/s11240014-0453-0

Ma, Y., Szostkiewicz, I., Korte, A., Moes, D., Yang, Y., Christmann, A., et al. (2009). Regulators of PP2C phosphatase activity function as abscisic acid sensors. Sci. (80-.) 324, 1064-1069. doi: 10.1126/science.1172408

Orman-Ligeza, B., Morris, E. C., Parizot, B., Lavigne, T., Babé, A., Ligeza, A., et al. (2018). The xerobranching response represses lateral root formation when roots are not in contact with water. Curr. Biol. 28, 3165-3173.e5. doi: 10.1016/ j.cub.2018.07.074

Pandey, S., Wang, R. S., Wilson, L., Li, S., Zhao, Z., Gookin, T. E., et al. (2010). Boolean modeling of transcriptome data reveals novel modes of heterotrimeric G-protein action. Mol. Syst. Biol. 6, 1-17. doi: 10.1038/msb.2010.28

Park, S. Y., Fung, P., Nishimura, N., Jensen, D. R., Fujii, H., Zhao, Y., et al. (2009). Abscisic acid inhibits type $2 \mathrm{C}$ protein phosphatases via the PYR/PYL family of START proteins. Sci. (80-.) 324, 1068-1071. doi: 10.1126/science.1173041

Pfaffl, M. W., Graham, W., and Horgan, L. D. (2002). Relative expression software tool (REST(C)) for group-wise comparison and statistical analysis of relative expression results in real-time PCR. Nucleic Acids Res. 30, 36e- 36. doi: 10.1093/nar/30.9.e36

Rasheed, S., Bashir, K., Matsui, A., Tanaka, M., and Seki, M. (2016). Transcriptomic analysis of soil-grown Arabidopsis thaliana roots and shoots in response to a drought stress. Front. Plant Sci. 7, 180. doi: 10.3389/ fpls.2016.00180

Schiml, S., Fauser, F., and Puchta, H. (2016). CRISPR/Cas-mediated site-specific mutagenesis in Arabidopsis thaliana using Cas9 nucleases and paired Nickases. Methods Mol. Biol. 1469, 110-122. doi: 10.1007/978-1-4939-4931-1_8

Schindelin, J., Arganda-Carrera, I., Frise, E., Verena, K., Mark, L., Tobias, P., et al. (2012). Fiji - an Open platform for biological image analysis. Nat. Methods 9, 676. doi: 10.1038/nmeth.2019.Fiji

Tischer, S. V., Wunschel, C., Papacek, M., Kleigrewe, K., Hofmann, T., Christmann, A., et al. (2017). Combinatorial interaction network of abscisic acid receptors and coreceptors from Arabidopsis thaliana. Proc. Natl. Acad. Sci. U.S.A. 114, 10280-10285. doi: 10.1073/pnas.1706593114
Ullah, A., Sun, H., Yang, X., and Zhang, X. (2017). Drought coping strategies in cotton: increased crop per drop. Plant Biotechnol. J. 15, 271-284. doi: 10.1111/ pbi. 12688

Van Der Weele, C. M., Spollen, W. G., Sharp, R. E., and Baskin, T. I. (2000). Growth of Arabidopsis thaliana seedlings under water deficit studied by control of water potential in nutrient-agar media. J. Exp. Bot. 51, 1555-1562. doi: 10.1093/jexbot/51.350.1555

Van Holle, S., and Van Damme, E. J. M. (2018). Signaling through plant lectins: modulation of plant immunity and beyond. Biochem. Soc Trans. 46, 217-233. doi: $10.1042 /$ bst20170371

Van Hove, J., Fouquaert, E., Smith, D. F., Proost, P., and Van Damme, E. J. M. (2011). Lectin activity of the nucleocytoplasmic EUL protein from Arabidopsis thaliana. Biochem. Biophys. Res. Commun. 414, 101-105. doi: 10.1016/ j.bbrc.2011.09.031

Van Hove, J., Stefanowicz, K., De Schutter, K., Eggermont, L., Lannoo, N., Al Atalah, B., et al. (2014). Transcriptional profiling of the lectin ArathEULS3 from Arabidopsis thaliana toward abiotic stresses. J. Plant Physiol. 171, 17631773. doi: 10.1016/j.jplph.2014.08.009

Van Hove, J., De Jaeger, G., De Winne, N., Guisez, Y., and Van Damme, E. J. M. (2015). The Arabidopsis lectin EULS3 is involved in stomatal closure. Plant Sci. 238, 312-322. doi: 10.1016/j.plantsci.2015.07.005

Vilches-Barro, A., and Maizel, A. (2015). Talking through walls: Mechanisms of lateral root emergence in Arabidopsis thaliana. Curr. Opin. Plant Biol. 23, 3138. doi: 10.1016/j.pbi.2014.10.005

Wang, R. S., Pandey, S., Li, S., Gookin, T. E., Zhao, Z., Albert, R., et al. (2011). Common and unique elements of the ABA-regulated transcriptome of Arabidopsis guard cells. BMC Genomics 12, 216. doi: 10.1186/1471-2164-12-216

Xie, K., Zhang, J., and Yang, Y. (2014). Genome-wide prediction of highly specific guide RNA spacers for CRISPR-Cas9-mediated genome editing in model plants and major crops. Mol. Plant 7, 923-926. doi: 10.1093/mp/ssu009

Xing, L., Zhao, Y., Gao, J., Xiang, C., and Zhu, J. K. (2016). The ABA receptor PYL9 together with PYL8 plays an important role in regulating lateral root growth. Sci. Rep. 6, 1-13. doi: 10.1038/srep27177

Yoshida, T., Christmann, A., Yamaguchi-Shinozaki, K., Grill, E., and Fernie, A. R. (2019). Revisiting the basal role of ABA - roles outside of stress. Trends Plant Sci. 24, 625-635. doi: 10.1016/j.tplants.2019.04.008

Conflict of Interest: The authors declare that the research was conducted in the absence of any commercial or financial relationships that could be construed as a potential conflict of interest.

Copyright (c) 2020 Dubiel, Beeckman, Smagghe and Van Damme. This is an openaccess article distributed under the terms of the Creative Commons Attribution License (CC BY). The use, distribution or reproduction in other forums is permitted, provided the original author(s) and the copyright owner(s) are credited and that the original publication in this journal is cited, in accordance with accepted academic practice. No use, distribution or reproduction is permitted which does not comply with these terms. 